\title{
Science of Mantras: A Hypothesis
}

\author{
Aditya Basu* \\ DR. D.Y. Patil Medical College, Kolhapur, Maharashtra, India
}

${ }^{\star}$ Correspondence to: Aditya Basu; DR. D.Y. Patil Medical College, Kolhapur, Maharashtra, India; Email: abasu9823@gmail.com

Received: Nov 13 ${ }^{\text {th }}, 2020$; Accepted: Dec $4^{\text {th }}, 2020$; Published: Dec $7^{\text {th }}, 2020$

Citation: Basu A. Science of Mantras: A Hypothesis. Gen Surg Open A Open J. 2020; I(3): 50-51. doi: 10.33169/surg.GSOAOJ-I-113

\begin{abstract}
This article Is about why practising Vedic mantras should be inculcated among people and that it alters the neural circuits of the brain.
\end{abstract}

Keywords: Neuroscience and religion; Synaptic plasticity; Sensory transduction .

\section{INTRODUCTION}

From the dawn of civilization Vedic and Buddhist chants have been seen to have a profound impact on the thinking and cognitive functions of human beings. With advancement of age it began to be regarded as "unscientific". Eventually these lost its glory and importance. These are not as 'unscientific' as it seems to be. In this article it has been hypothesized that practicing these regularly would actually have a profound impact on our mental abilities.

\section{CORE CONCEPTS}

\section{Sensory Transduction}

Sensory transduction is the process by which the external stimuli is converted into action potentials in cells. It is not new to the us that sensory receptors have mechanical stimuli sensitive channels. For example- Paccinian corpuscles have nerve endings encapsulated with many layers of Schwann cell and surrounded by a buffered extracellular fluid. These endings have $\mathrm{Na}+$ channels. Mechanical forces leads to the opening of channels and this leads to depolarization and generation of action potentials.

\section{Synaptic Plasticity}

Plasticity is the capacity of the nervous system to change. Plasticity in our nervous systems can occur at synapses, within neurons, within glial cell. It affects the structure and function of neural circuits and systems and it forms the basis of memory, motor skills, cognitive skills, injury and adaptation.

Mechanism of plasticity: Earlier experiments were performed on hippocampus of rodents where the effect of tetanic stimulation and intermittent stimulation on Excitatory Post Synaptic Potential (epsp) was found.
It was seen that the amplitude of epsp in tetanic stimulation increased with time; but intermittent firing did not let to change in the amplitude of epsp. This difference in amplitude is called long term potentiation(LTP). Thus repeated stimulation "strengthened the synapse".(Figure 1)

Figure 1: Image of LTP: brain.mcgill.ca. ${ }^{1}$

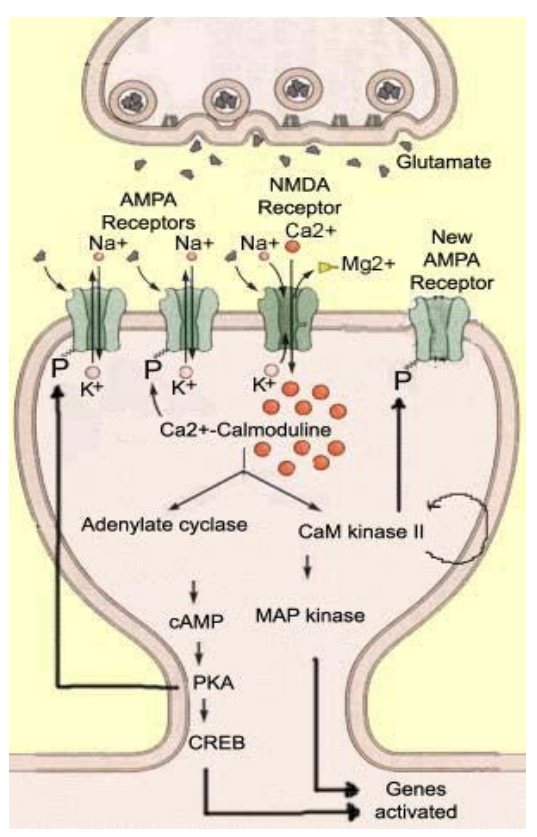

AMPA and NMDA play an important role in plasticity. Binding of glutamate to AMPA leads to opening of $\mathrm{Na}+$ channels and as a 
result it comes in. But NMDA receptors have a magnesium ion binding site which prevents entry of ions through their pore.

However if their is strong depolarizing potential in the presynaptic neuron it leads to the flooding of glutamate in the synapse. This leads to the increased binding of glutamate to AMPA and thus increased sodium ions enter the post synaptic neuron. This increased conc. Of $\mathrm{Na}+$ repels and passively removes the Magnesium ion from the NMDA receptor. When this occurs, NMDA allows $\mathrm{Na}+$ and $\mathrm{Ca}+2$ ions to flow in. This increased Calcium causes the secondary activation of messengers (like protein kinases which phosphorylates proteins)

Protein kinase A phosphorylates AMPA receptors and keep them open for a longer period of time allowing further depolarization. CREB protein activation has lead to the development of AMPA receptors. The intra neuronal Calcium binds with Calmodulin and activates CAM kinase 2. These not only phosphorylate AMPA receptors but also activate protein like MAP kinases which are involved in building dendrites and NMDA receptors themselves.

All these events lead to the increase in amplitude of epsp in the neuron.

Now in the same rat model low frequency of stimulation was given via microelectrodes.

The amplitude of epsp decreases: This low frequency causes depolarization of post synaptic neuron but it causes a decreased or slower levels of increased calcium which instead of activating protein kinases, activates phosphatases which dephosphorylates proteins and leads to internalization of AMPA receptors. Hence amplitude of epsp decreases with time. This is called Long Term Depression(LTD).

Spike time dependent plasticity: Now we know that it is difficult for our brain to recieve such train of tetanic impulses but we do LTP and LTD in neurons. How is this possible?

Experiments were carried out where supplying a spike of action potential just before the actual AP arises at the synapse leads to increased amplitude of epsp in post synaptic neuron. And whereas if we give a spike of AP some milliseconds later after the actual AP has arrived at the synapse we see that there is a decrease in amplitude of the post synaptic neuron. This is called spike time dependent plasticity and it explains plasticity framework of our brain.

\section{DISCUSSION}

Our vocal cords produce vibrations which are transmitted to the surrounding tissues. Louder the sound produced by vocal cords the more is the vibration that is transmitted to the surrounding tissues. This mechanical energy transduces into the receptors as action potential much the same way as the peripheral receptors on other parts of the body. These receptors may be present in the upper proximity of our body or in the scalp. The auditory receptors may also be involved in transducing these vibrations. Hence following repeated activation the above mechanisms of Long Term potentiation and Long term depression occur. This in turn affects gene transcription and production of proteins. Which in turn will have an effect on the cognitive functions.

I would like to further expand my idea. Religious mantras are chanted out a fixed number of times or in multiples of a particular number. This may be thought of as a repeated stimulation of neurons which leads to increase or decrease in the strength of synapse depending whether the stimulus was given before or after the action potential.

\section{CONCLUSION}

Vedic mantras would play and important role in altering the cognitive functions of human beings.

\section{REFERENCES}

1. brain.mcgill.ca

2. Neuroscience textbook by Dale Purves 